\title{
A Novel Approach for Creating Consistent Trust and Cooperation (CTC) among Mobile Nodes of Ad Hoc Network
}

\author{
Khurram S.Rajput, Khaled M. Elleithy, Syed S. Rizvi \\ Computer Science and Engineering Department \\ University of Bridgeport \\ Bridgeport, CT 06605 \\ \{krajput, srizvi, elleithy\}@bridgeport.edu
}

\begin{abstract}
This paper provides a critical analysis of the recent research wok and its impact on the overall performance of a mobile Ad hoc network. In this paper, we clarify some of the misconceptions in the understating of selfishness and missbehavior of nodes. Moreover, we propose a mathematical model that based on the time division technique to minimize the node misbehavior by avoiding unnecessary elimination of bad nodes. Our proposed approach not only improves the resource sharing but also creates a consistent trust and cooperation (CTC) environment among the mobile nodes. We believe, that the proposed mathematical model not only points out the weaknesses of the recent research work but also approximates the optimal values of the critical parameters such as throughput, transmission over head, channel capacity etc. The simulation results demonstrate the success of the proposed approach that significantly minimizes the malicious nodes and consequently maximizes the overall throughput of the Ad Hoc network than the other well known schemes.
\end{abstract}

\section{INTRODUCTION}

Misbehavior in mobile ad-hoc networks occurs for several reasons. Selfish nodes misbehave to save power or to improve their access to service relative to others [1]. Malicious intentions result in misbehavior as exemplified by denial of service attacks. Faulty nodes simply misbehave accidentally. Regardless of the motivation for misbehavior its impact on the mobile ad-hoc network proves to be detrimental, decreasing the performance and the fairness of the network, and in the extreme case, resulting in a non-functional network [2]. This paper addresses the question of how to make network functional for normal nodes when other nodes do not route and forward packets correctly. Specifically, in mobile ad-hoc networks, nodes do not rely on any routing infrastructure but relay on packets for each other. Thus communication in mobile ad-hoc networks functions properly only if the participating nodes cooperate in routing and forwarding. However, it may be advantageous for in nodes not to cooperate, such as a selfish node wants to preserve own resource to save power, memory, network-bandwidth, and local CPU time. Therefore nodes assume themselves that other nodes would forward the packet. This selfish or malicious intention of nodes can significantly degrade the performance of mobile ad-hoc-networks by denial of service.

In this paper, we focus on the design of a new time division based scheme that can avoid unnecessary elimination of malicious nodes while at the same time maximize the throughput of the system by increasing the recourse sharing among the mobile nodes. The existing methods/algorithms not only creating a performance bottleneck (i.e., by increasing the network congestion, transmission overhead etc.) but also diminishing the self-growing characteristic of a peer to peer network. These methods such as CONFIDANT [3] and CORE [4] force the participating nodes to adopt the same behavior as the other selfish nodes that have already been removed from the network due to the lack of resources. We believe that we should not propose any algorithm/method that becomes the reason for reducing the network resources and consequently force the existing participating nodes to behave exactly in the same way as other removed nodes. Instead, we strongly believe that we should come up with something that not only improves the resources and resource sharing but also creates a consistent trust and cooperation (CTC) environment among the mobile nodes.

The rest of this paper is organized as follows: Section II describes the research that has already been done in this area. The proposed analytical and mathematical models for CTC are presented in Section III. The simulation results are provided in section IV. Finally, section V concludes the paper.

\section{Related Work}

The terms reputation and trust are being used for various concepts in the literature, also synonymously $[5,6]$. We define the term reputation here to mean the performance of a principal in participating in the base protocol as seen by others. The key thing in reputation system is watchdog and pathrater which have been proposed by Marti, Giuli, Lai and Baker [7]. They observed increased throughput in mobile adhoc networks by complementing DSR with a watchdog for detection of denied packet forwarding and a path rater for 
trust management and routing policy rating every path used, which enable nodes to avoid malicious nodes in their routes as a reaction. Their approach does not punish malicious nodes that do not cooperate, but rather relieves them of the burden of forwarding for others, whereas their messages are forwarded without complaint. This way, the malicious nodes are rewarded and reinforced in their behavior. They used a watchdog that identifies misbehaving nodes and a pathrater that helps routing protocols avoid these nodes. When used together in a network with moderate mobility, the two techniques increase throughput by $17 \%$ in the presence of $40 \%$ misbehaving nodes, while increasing the percentage of overhead transmissions from the standard routing protocol's $9 \%$ to $17 \%$. During extreme mobility, watchdog and pathrater can increase network throughput by $27 \%$, while increasing the overhead transmissions from the standard routing protocol's $12 \%$ to $24 \%$.

CORE, a collaborative reputation mechanism proposed by Michiardi and Molva [4], also has a watchdog component; however it is complemented by a reputation mechanism that differentiates between subjective reputation (observations), indirect reputation (positive reports by others), and functional reputation (task-specific behavior), which are weighted for a combined reputation value that is used to make decisions about cooperation or gradual isolation of a node.

\section{The Proposed Analytical And Mathematical Model For Creating Consistent Trust And Cooperation (CTC)}

The creation of mathematical model can be viewed as a formalization of the proposed hypothesis. Based on the proposed mathematical model, we perform the numerical and simulation analysis for variety of scenarios in two parts. First, we use the mathematical model to run different scenarios in order to determine the performance of Ad-hoc networks by analyzing different critical network parameters such as throughput, transmission overhead and the utilization. Secondly, we use the same set of parameters as a performance measure.

\section{A. The Proposed Analytical Model}

We model the Ad-hoc network in much the same way as other researcher does except this paper introduces the new concept of time division. The idea of time division can simply be envisioned by considering a particular node of a network that has a potential to misbehave in the absence of the sufficient resources require to forward the packets of the neighboring nodes. This implies that if one can ensure that the network has enough resources that can be shared equally among the network nodes, then it can be assumed that the possibility of node misbehavior degrades significantly. Thus this reduction in the node misbehavior can be achieved through the time division technique that divides the time asymmetrically into the following two times: transmission-time required for node-packets and transmission-time required for neighbor-packets. The asymmetric division enables a node to effectively adjust the time required to transmit its own packets and/or the neighbor's packets. The reason for using the asymmetric division of the available time is to allow a node to effectively utilize the time by dividing it with respect to its current status (i.e., the available recourses) and consequently utilizing the bandwidth in an efficient manner. The efficient utilization of the bandwidth satisfies the requirement of the fairness which is one of the key factors that forces a node to unfair with its neighbor. This indirectly points that we reduce the chances of misbehave since the node now has a total authority on the available resources. It should also be noted that we adopt an asymmetric approach to work with the time division method for this research which opposed to the conventional division of time (i.e., the symmetric or equal division employed by many different techniques). In other words, the proposed method optimizes the performance by effectively reducing the chances of node misbehave at the expense of comparatively complex logic.

\section{B. The Proposed Mathematical Model}

Before going to develop the actual mathematical model based on the above analytical model, it is worth mentioning some of our key assumptions. These assumptions help understanding the complex relationship between a large numbers of parameters. For the proposed mathematical model, we assume that a system has $K$ nodes where each individual node $k$ not only works as a normal mobile station but also works as a packet forwarding device for the other nodes. In addition, we assume that any kind of topology can be implemented among the mobile nodes to construct the Ad-hoc network. For the ease of simplicity, we perform the numerical analysis for a single node $k$. This can be further extended for the whole network by computing the collective behavior of the Ad-hoc network.

The primary principal of Ad-hoc network is that it allows each node of the network to fully participate in the construction of the network. The word fully participation leads us to the fact that a node not only transmits its own packets to the other neighboring nodes but also provides its services to other nodes as a forwarding device. For the proposed method, we assume that a node can decide to transmit its own packets with a certain probability while at the same time it can also deny the transmission of the other neighboring packets with a difference of a certain probabilities. In simple words, we can develop a relationship between these two probabilities as follows: a node can transmit the self generated packet(s) with a probability of $p$ where as it can transmit its neighbor packet( $(s)$ with the probability of $q$.

Suppose, $p$ is the probability for which a node forwards personal packets where as $p(I-p)$ is the probability for which a node transmit packets received from one ore more neighbors. In addition, we assume that $k$ is total number of packets that can be transmitted by a certain node of the Ad-hoc network. The total numbers of packets include both the self generated packets and the packets receive from one or more nodes. Taking this into account, we can say that if the probability of transmission of a single packet is $(1-p)^{x}$ where $x$ represents a single packet, then the probability to transmission $k$ packets would be $(1-p)^{k}$ where $k$ represents the total number of packets 
that a node can transmit. This leads us to the following mathematical fact:

$$
(1-p)^{k}
$$

Equation (1) can simply be formalized for $k$ number of packets as follows:

$$
p(1-p)^{k}
$$

As mentioned earlier, the proposed method is exclusively dependent on the time division methodology where a node can divide the time asymmetrically to represent the time it needs to transmit self generated packets as well as the time it takes to transit the packets arriving from one or more nodes. To make our proposed approach more realistic, we assume that if the packet that resides in a certain node is not delivered to its intended destination within the specified time, then that packet must be discarded by the node. The lost of the packet at the node level forces us to retransmit the packet. For the ease of understating, we assume that the time a node takes to transmit self generated packet can be represented as $t_{p p}$ where as the time it takes to forward the packets received from one or more neighbors is represented as $t_{n p}$. It should be noted that the total available time per node is just the sum of the time a node takes to transmit self generated packet and time it takes to forward the packets received from one or more neighbors. This relationship can be mathematically expressed in the following equation:

$$
t_{i}=t_{p p}+t_{n p}
$$

where $i$ represents the index of node that can be expended from 1 to $K$ (i.e., $K$ represents the total nodes present in a Adhoc network)

The maximum throughput is defined as the asymptotic throughput when the load is very large. In packet switched network where the load and the throughput are equal, the maximum throughput may be defined as the load in bits per seconds. Thus this in turns lead us to a fact that the maximum throughput can not be defined in the presence of packet drops at the node level. As mentioned earlier, to make our model more realistic we consider the possibility of packet drops and consequently the packet retransmission at the node level. The throughput from the proposed algorithm for a certain node of the Ad hoc network can be computed as follows:

$$
T_{p u t}=\text { Total Packets Forwarded/Total Time }
$$

The denominator of (4) is derived from (3) where as the numerator of equation is determined by using (1) and (2). One can see that as we increase the left hand side of (2), it causes a decrease in the left hand side of (4). It should also be noted that as we increase the sum of (1) and (2), it significantly increases the left hand side of (4). To make these relationships simple, we can say that the increase in the sum of (1) and (2) causes an increase in the throughput where as an increase in the total time that is determined by (3) causes a decrease in the throughput per node. This is because the more we increase the time, the more bandwidth we need to reserve to satisfy the transmission requirements.

A significant increase in the bandwidth utilization (which is beyond the scope of the available bandwidth per node) represents degradation in the throughput that indicates an increase in the possibility of node misbehavior. Thus, this implies that the proposed algorithm is not only improving the performance but also providing a chance to choose the optimal values of critical parameters. Equation (4) can be further simplified in the following form:

$$
T_{\text {put }}=\frac{\text { Node's Packets }+ \text { Neighbour's Packets }}{\text { Total Time }}
$$

To formalize the above discussion, we can combine probabilities of transmission from (1) and (2) with the total available time per node from (3) in (5). Thus this expresses the node throughput not only by means of total available time but also by means of the total number of packets a node can transmit. The final result can be expressed in the following equation:

$$
T_{p u t}=(1-p)^{k}+(1-p)^{k} / t_{i}
$$

It should be noted that (6) gives node throughput by considering the time $t_{i}$ spends on a single packet (that is the time spend on one packet is the sum of the time spend on self generated packets and the neighbor packets). Solving (5) for $k$ number of packets in terms of the total time required by a node can be expressed in the following equation:

$$
t_{i}=\sum_{1}^{k} t_{p p(k)}+\sum_{1}^{k} t_{n p(k)} \quad 1 \leq k<\infty
$$

where $k$ in (7) represents the number of packets that are bounded between 1 and the infinity. The first and the second quantity of the right hand side of (7) are indicating the time required transmitting the self generated packets and the time required to transmit the neighbor packets. The generic time equation can simply be stated as:

$$
t=\text { no of packet/data rate }
$$

Using (8), one can now compute the two major components of the proposed time division algorithm. It is essential in order to understand the concept of asymmetric division. One of the two asymmetric time division quantities can be quantified as follows:

$$
t_{n p}=P(1-P)^{k} / D_{R}
$$

where $D_{R}$ in (9) represents the data rate.

Recall one of our fundamental assumptions that a node transmits $k$ number of packets in total time $t_{i}$. This assumption allows us to set up a lower and upper bound on the number of packets that a node can transmit. Therefore, the limit for $k$ should exist somewhere zero to infinity. One of the main reasons for recalling this assumption is make a more generalized form of (9). Taking these two factors into account, one can generalize (9) as follows: 


$$
t_{n p}=\sum_{k \geq 1}^{k \leq \infty} \frac{P(1-P)^{k}}{D_{R}} \quad \text { where } 1 \leq K \leq \infty
$$

The numerator of (10) is just a summation of total packets forwarded by a node with respect to the probabilities set up at static time. If $t_{p p}$ is the total time taken by a node to forward its own $k$ number ofpackets, then equation for $t_{p p}$ can be rewritten as.

$$
t_{p p}=\sum_{1}^{k}\left\{\frac{(1-P)^{k}}{D_{R}}\right\} \quad \text { where } 1 \leq K \leq \infty
$$

Equation (11) is the summation of probabilities of one packet to $\mathrm{k}$ number of packets per node in the presence of a certain data rate. By substituting the value of total time $t_{i}$ from (3) into (6), we get

$$
T_{p u t}=\left\{(1-p)^{k}+p(1-p)^{k}\right\} /\left\{t_{p p}+t_{n p}\right\}
$$

In order to generalize (12), we need to substitute the values of $t_{p p}$ and $t_{n p}$ from (10) and (11), respectively, into (12), we get:

$$
T_{p u t}=\sum_{1}^{k}\left[\frac{D_{R}\left\{(1-p)^{k}+p(1-p)^{k}\right\}}{(1-p)^{k}+p(1-p)^{k}}\right]
$$

The first two quantities in denominator of (13) represent the summation of the time a node takes to transmit the personal packet and the neighbor's packets. It should be noted that (13) is generalized in a sense that it accommodates $\mathrm{k}$ number of packets that a node can deal at a certain point of time. To make it simple, we can rewrite equation as follows:

$$
T(\text { put of node })=\sum_{1}^{k}\left[\frac{D_{R} \times(1-p)^{k}+p(1-p)^{k} \times D_{R}}{\left\{(1-p)^{k}+p(1-p)^{k}\right\}}\right]
$$

Equation (14) is the total throughput of a node for $k$ number of packets that a node can transmit. Let us assume that $N p$ is the power of node and $K$ is the number of packet that a node can transmit. Taking these assumptions into account, one can derive a generic expression for utilization as follows:

$$
U=N_{\text {pout }} / N_{\text {pin }}
$$

We call (15) as a generic mathematical expression of utilization, since both the numerator and the denominator are unknown and need to be determined to find out a more specific expression. Therefore, this new concept of power division leads us to the following mathematical expression for node-utilization with respect to the node's personal packets.

$$
N_{\text {ppout }}=\sum_{1}^{K}\left\{K_{\text {Pout }} / t_{p p}\right\}
$$

It should be noted that (16) is a more specific form of (15) since it only account for the personal packets. Thus the opposite hypothesis leads us to the following mathematical expression for the node utilization with respect to the personal packets:

$$
N_{\text {pnout }}=\sum_{K \geq 1}^{K<\infty}\left\{K_{\text {nout }} / t_{n p}\right\}
$$

Contrary to (17), there should be an equivalent possibility of node inputs that can easily be computed as follows:

$$
N_{\text {pnin }}=\sum_{1}^{K}\left(K_{\text {nin }} / t_{n p}\right)
$$

It should be noted that (18) can be useful to compute the output of the nodes in terms of the inputs of the node. In other words $N_{p_{\text {out }}}$ is the sum of work on outgoing personal and neighbor packets that lead us to derive the simple mathematical relationship:

$$
N_{\text {pout }}=N_{\text {pp (out })}+N_{\text {pnout }}
$$

In order to show that (19) is a valid true mathematical relationship between the input and output lines of a node, one needs to give another relationship as follows:

$$
N_{\text {pin }}=N_{\text {pnin }}
$$

This should now be clear that one of the reasons for deriving the above two relationship is to derive a more general expression from (16) and (17). Therefore, by substituting (16) and (17) into (19), we get the following equation:

$$
N_{\text {ppout }}=\sum_{k \geq 1}^{k<\infty}\left[\left\{\frac{K_{\text {ppout }}}{t_{\text {pp }}}\right\}+\left\{\frac{K_{\text {nout }}}{t_{n p}}\right\}\right]
$$

Similarly, we can derive another expression using (20) which opposed to (21) as follows:

$$
N_{P i n}=\sum_{1}^{K}\left(\frac{K_{n i n}}{t_{n p}}\right)
$$

The last two equations (i.e., (21) and (22)) can now be used to derive the final expression for utilization as follows:

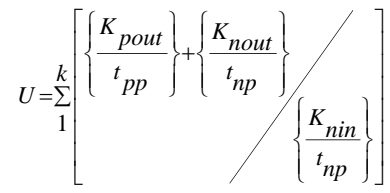

All lines that are used for transferring the data or packets are also used for receiving the data or packets from neighbor nodes. This implies that the utilization per channel or line can be computed using (23). If we denote this line-utilization as (24), we can extend it to generalized (23). 


$$
U_{R}=\sum_{k \geq 1}^{k<\infty} \frac{\left(K_{\text {pout }} / K_{\text {nout }}\right) t_{n p}}{K_{n i n}}
$$

If we assume that $n$ numbers of routes are attached through the targeted node, then the utilization of the targeted node on all routes can simply be computed by summing the utilization of each node per channel. This can lead us to the following equation:

$$
U_{t}=\sum_{n \geq 1}^{n<\infty} U_{R_{n}} \quad \text { where } 1 \leq n<\infty
$$

This can also be interpreted as follows:

$$
U_{t}=U_{R 1}+U_{R 2}+U_{R 3}+\ldots \ldots . .+U_{R n}
$$

Therefore, the total utilization of system can be derived from (23) and (25) as follows:

$$
U_{t}=\sum_{n \geq 1}^{n<\infty} \sum_{k \geq 1}^{k<\infty} \frac{\left(K_{\text {pout }} / t_{p p}\right)+\left(K_{\text {nout }} / t_{n p}\right)}{K_{\text {nin }} / t_{n p}}
$$

We perform some simplification in (27) that results the following equation:

$$
U_{t}=\sum_{n \geq 1}^{n<\infty} \sum_{k \geq 1}^{k<\infty} \frac{1}{K_{\text {nin }}}\left[K_{\text {pout }}\left(t_{n p} / t_{p p}\right)+K_{\text {nout }}\right]
$$

The above equation can be used to compute the total utilization of a certain node for all packets that it can forward and/or receive from one of its neighbor though all possible channels.

\section{THE EXPERIMENTAL VERIFICATION AND THE PERFORMANCE ANALYSIS OF THE CTC}

We have shown that the system throughput can be measured in term of packets that neighboring node is generated as well as the self generated packets. To make the proposed methodology up to the standard, we derive the formula for computing the packet drop per node using (5). As mentioned earlier, we determine the behavior of the malicious node in terms of the number of packets that should have transmitted to the intended destination. For taking this into account, one can say that the effective throughput of a node is entirely dependence on how efficiently the node is forwarding the neighbor packets and thus creating a consistent trust environment among the nodes.

\section{A. Case I}

For case-1, we assume that the self generated packets per node are constant. We assume that one of the neighboring nodes of the target node sends packets at a certain rate that

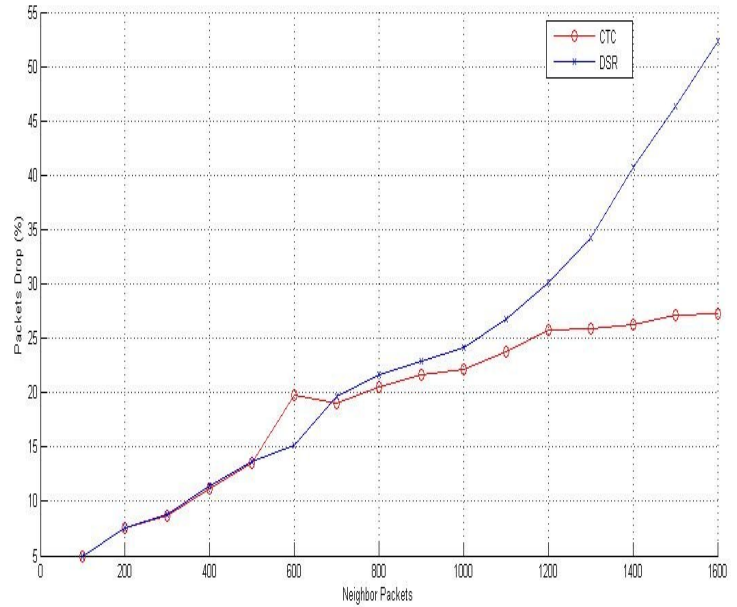

Figure 1: Neighbor packet generation vs. packet drop

will increase linearly over the total simulation time. This assumption helps understanding the true performance of the proposed CTC algorithm. Fig. 1 shows the simulation results of packet-drops per node with respect to the number of packets generated by one of the neighboring nodes. It should be noted that as we increase the self generated packets, the number of packet-drops per node is increased. In addition, it can be seen in Fig. 1 that for a small value of neighbor packet generation (typically 500), both CTC and DSR are overlapping each other. However a slight increase in the neighbor packet generation causes a performance difference between these two approaches.

\section{B. Case II}

CASE-II is different from CASE-I in such a way that both inputs of a node-forwarding system become a linear function of the node-time. The simulation result of this case satisfies the proposed mathematical model discussed in Section III in a way that the overall packet drop performance of both

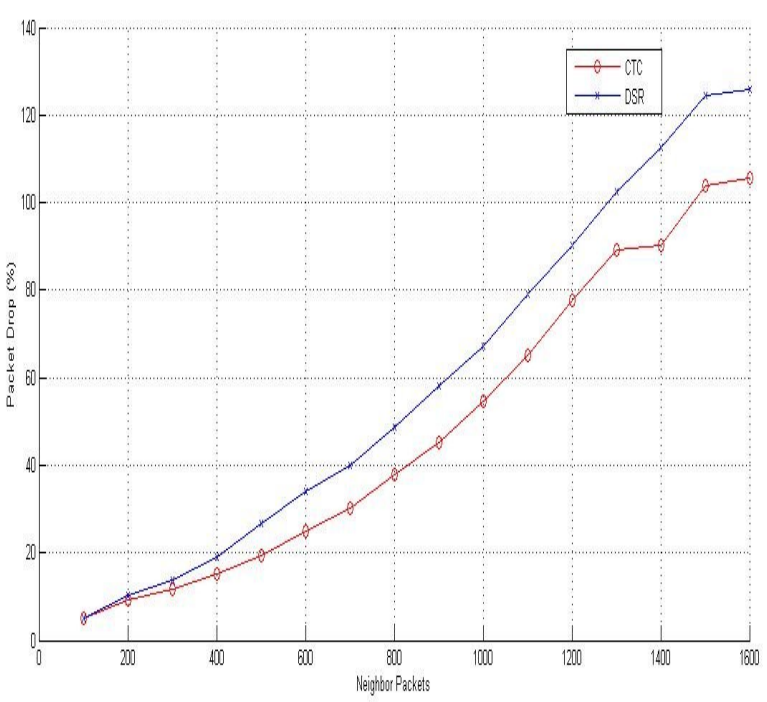

Figure 2: Neighbor packet generation vs. packet drop 
investigated algorithms decreases. It can be seen that the packet drop is more rapid in Fig. 2 with respect to the neighbor-generated packets. In harmony with our expectations, as the number of neighbor-generated packets increased, the packet-drop performance of the proposed algorithm degraded. However, the performance degradation of the proposed algorithm was small compared to the performance degradation of the DSR algorithms.

\section{Case III}

The parameters-assumption for CASE-III is different from the previous cases in such a way that now one input (that is the neighbor-generated packets) of a node-forwarding system becomes a linear increasing function of the node total time where as the input (that is the neighbor-generated packets) becomes a linear decreasing function of the node total time. The expected output of this simulation was exactly the same as we were expecting based on our proposed mathematical model. That is the values of packet-drop for both CTC and DSR decreases as compared to the other two cases we discussed above.

\section{Case IV}

For this case, we assume that the neighbor-generated packet is a constant function of time. On the other hand, we consider self-generated packets as a linear increasing function of the total node time. It should be noted that the term linear increase or decrease implies a constant uniform change in the system parameter with respect to time. This case can also be considered as a reciprocal of CASE-I from its fundamental assumptions point of view. Thus we should also expect a reciprocal output for this simulation.

\section{CONCLUSION}

This paper proposed both analytical and mathematical model that can be used to effectively reduce the number of malicious nodes and packet drops. Our simulation results

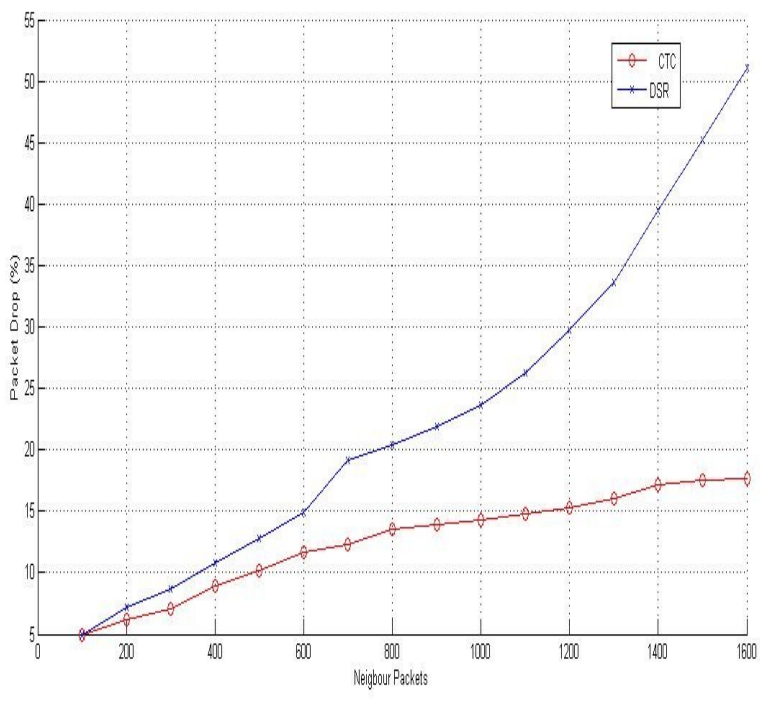

Figure 3: Neighbor packet generation vs. packet drop

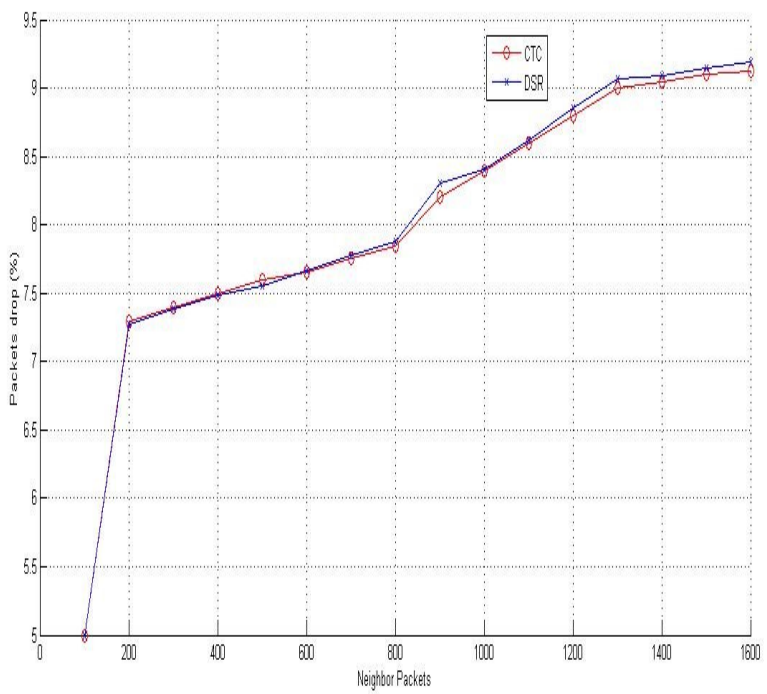

Figure 4: Neighbor packet generation vs. packet drop

demonstrated that the proposed mathematical model not only points out the weaknesses of the recent research work but also approximates the optimal values of the critical parameters. Simulation results presented in this paper show that how the performance of mobile Ad hoc networks degrades significantly when the nodes eliminations are frequent. The simulation results of this paper are completely based on the proposed mathematical model for both lightly and heavily loaded networks. These results addressed many critical system parameters such as packet drop and packet loss versus malicious nodes, neighbor packet generation and drop ratio, and throughput per node per system.

\section{REFERENCES}

[1] Zhang and W. Lee, "Intrusion Detection in wireless Ad-hoc networks," in Proceedings of MOBICOM 2000, pp. 275-283, 2000.

[2] Y. Huang, W. Fan, W. Lee, and P. Yu, "Cross-Feature analysis for detecting Ad-hoc routing Anomalies," in Proceedings of the 23rd International Conference on Distributed Computing Systems (ICDCS 2003), Providence, RI, pp. 478-487, May 2003.

[3] S. Marti, T. Giuli, K. Lai, and M. Baker, "Mitigating routing Misbehavior in mobile Ad hoc networks," in Proceedings of MOBICOM 2000, pp. 255-265, 2000.

[4] P. Michiardi and R. Molva, "CORE: A Collaborative Reputation Mechanism to enforce node Cooperation in mobile Ad hoc networks," Sixth IFIP conference on security communications, and multimedia (CMS 2002), Portoroz, Slovenia, 2002.

[5] T. Moreton and A. Twigg, "Enforcing collaboration in P2P routing services," 2003.

[6] S. Bansal and M. Baker, "Observation-based Cooperation Enforcement in Ad hoc networks," Technical Report, 2003.

[7] S. Marti, T. Giuli, K. Lai, and M. Baker, "Mitigating routing Misbehavior in mobile Ad hoc networks," in Proceedings of MOBICOM 2000, pp. 255-265, 2000. 